\title{
SUCO DE CAJÁ (Spondias mombin L.) COMO ADJUNTO NA PRODUÇÃO DE CERVEJA: ANÁLISES FISÍCO-QUÍMICAS, SENSORIAL E ESTUDO CINÉTICO.
}

\author{
L. D. MUNIZ ${ }^{1}$, V. F. de MELO ${ }^{1}$, J. A. C. BISPO ${ }^{1}$, V. L. D. BIANCHI ${ }^{2}$, G. B. M. de CARVALHO ${ }^{1}$ \\ ${ }^{1}$ Universidade Estadual de Feira de Santana, Departamento de Tecnologia \\ ${ }^{2}$ Universidade Estadual Paulista Júlio de Mesquita Filho, Departamento de Engenharia e Tecnologia \\ de Alimentos
}

E-mail para contato: giovanibrandao.uefs@gmail.com

\begin{abstract}
RESUMO - O presente trabalho aborda a aplicação de adjunto misto de suco de cajá e sacarose em substituição a $15 \%$ do malte de cevada na produção de uma cerveja Pilsen, em escala piloto de 60 litros. O acompanhamento físico-químico e cinético foi realizado durante as fases de fermentação e maturação. No ponto final a cerveja apresentou 5,51\% de etanol (v/v), com o consumo de $97,01 \mathrm{~g} / \mathrm{L}$ de extrato aparente, resultando em uma fermentabilidade de $73,49 \%$. A cerveja alcançou a produtividade volumétrica (Qp) de 1,07 g/L.h em 12 horas de fermentação e o maior valor de Yp/s nessas condições foi de $0,50 \mathrm{~g} / \mathrm{g}$ em 50 horas. Os valores máximos de $\mu \mathrm{x}, \mu \mathrm{s}$ e $\mu \mathrm{p}\left(0,134 \mathrm{~h}^{-1}, 2,95 \mathrm{gs} / \mathrm{gx}\right.$.h e 1,00 gp/gx.h) foram alcançados logo nas primeiras horas da fermentação primária e o tempo de geração da levedura foi de 5,17 horas. $85 \%$ dos provadores na análise sensorial demonstraram gostar da cerveja de cajá, sendo que dos 60 avaliadores presentes, 87\% apresentaram interesse na compra do produto.
\end{abstract}

\section{INTRODUÇÃO}

No cenário mundial atual, as indústrias precisam ser mais competitivas oferecendo produtos diferenciados para os mais diversificados consumidores. Esse perfil tem gerado um grande interesse pelas indústrias em procurar novos processos, tecnologias e novos produtos para atender às tendências inovadoras.

A indústria cervejeira não fica atrás nesse mercado e vem utilizando novas técnicas no processamento e investindo em pesquisas de novos ingredientes com o objetivo de atender cada vez mais aos consumidores mais exigentes e reduzir custos na produção, ganhando espaço no mercado.

Utilizam-se os adjuntos especialmente por questões econômicas, pois, apresentam menor custo na produção de extrato. Além disso, melhoram a qualidade sensorial e físico-química da cerveja acabada. Podem ser considerados como diluidores de todos os componentes do mosto cervejeiro, exceto os açúcares. De fato, reduzem o teor de nitrogênio solúvel, ou melhor, proteína solúvel do mosto, diminuindo a ocorrência de infecção láctea na cerveja, melhorando a sua estabilidade coloidal e, consequentemente, aumentando a vida de prateleira do produto. As cervejas que utilizam adjunto em sua composição são mais refrescantes e leves, apresentam normalmente maior brilho e cor mais 
clara. Há perspectivas de que fatores econômicos levem a um aumento na utilização de adjuntos pelas cervejarias em todo o mundo (VENTURINI FILHO, 2001).

O presente trabalho desenvolve a utilização do suco misto de Cajá (Spondias mombin L.) e sacarose como adjunto do malte na produção de uma cerveja tipo lager, substituindo $15 \%$ do mosto de puro malte pelo adjunto, em escala piloto. O acompanhamento analítico e cinético, através de análises físico-químicas, foi realizado a fim de caracterizar a cerveja obtida e uma análise sensorial foi realizada com o objetivo de identificar a aprovação do produto frente ao consumidor final.

\section{MATERIAL E MÉTODOS}

\subsection{Preparo do suco misto de cajá e sacarose}

O suco utilizado como adjunto foi produzido a partir de polpas comerciais da marca Brasfrut ${ }^{\circ}$, compradas no mercado local da cidade de Feira de Santana / BA - Brasil. Foram preparados 9 litros de suco na proporção de 1:1 em volume de água destilada, com o objetivo de se produzir 60 litros de cerveja com $15 \%$ de adjunto. O suco foi chaptalizado com sacarose comercial, de acordo com Cataluña (1988), tornando-o um adjunto misto de suco e açúcar, para o valor de $12{ }^{\circ}$ Brix. Após a chaptalização o pH do suco foi desacidificado com adição de $\mathrm{CaCO}_{3}$ para o valor de 5,0. Metabissulfito de potássio foi adicionado ao suco misto como antimicrobiano.

\subsection{Produção da cerveja}

O mosto cervejeiro foi preparado de acordo com as técnicas de fermentação convencionais (com as características finais de $12{ }^{\circ} \mathrm{Brix}$ e $\left.\mathrm{pH}=5,0\right)$ para o preparo de cervejas tipo Pilsen (CARVALHO et al,. 2008) nas instalações da cervejaria piloto, localizada no Departamento de Tecnologia da Universidade Estadual de Feira de Santana / BA - Brasil.

\subsection{Acompanhamento analítico da cerveja}

Durante toda a fermentação primária e secundária (maturação) amostras foram periodicamente retiradas (em tempos de 4 em 4 horas, nas primeiras 24 horas da fermentação, 12 em 12 durante toda a fermentação primária e 24 em 24 horas durante a maturação) para determinar a viabilidade e concentração celular, concentração de etanol, teor de extrato aparente, densidade, $\mathrm{pH}$, sólidos solúveis e açúcares redutores e totais. Desta forma, amostras de $20 \mathrm{~mL}$ de mosto foram incialmente desgaseificadas em balão volumétrico de $250 \mathrm{~mL}$ sob agitação manual e abertura do balão para a saída do $\mathrm{CO}_{2}$. Dos $20 \mathrm{~mL}$ retirados, $1 \mathrm{~mL}$ foi utilizado para a determinação da viabilidade celular e os outros $19 \mathrm{~mL}$ foram centrifugados em centrífuga de Eppendorf para a realização das demais análises.

\subsection{Determinação da concentração e viabilidade celular}

A concentração e viabilidade celular (células viáveis e não viáveis) foram determinadas pelo Método Internacional de coloração com azul de metileno segundo ASBC (1996) em contagem na câmara de Neubauer $\left(1 / 400 \mathrm{~mm}^{2}\right.$ x $\left.1 / 10 \mathrm{~mm}\right)$, expressas em cel/mL. 
A concentração celular ainda foi determinada através do peso seco das leveduras (expresso em $\mathrm{g} / \mathrm{L})$, onde amostras $(10 \mathrm{~mL}$ ) foram centrifugadas a 4000xg por $20 \mathrm{~min}$, recuperando-se a massa de células, que foi lavada e centrifugada a $13000 \mathrm{rpm}$ por $10 \mathrm{~min}$ com água destilada. A massa celular foi ressuspensa com água destilada e novamente centrifugada e então seca em estufa a $105{ }^{\circ} \mathrm{C}$ até peso constante em cadinho de porcelana.

\subsection{Determinação do extrato aparente, concentração de etanol e densidade}

O teor de extrato aparente $\left({ }^{\circ} \mathrm{P}\right)$, concentração de etanol $(\% \mathrm{v} / \mathrm{v})$ e a densidade $(\mathrm{g} / \mathrm{mL})$ foram determinados através do densímetro de bancada (Rudolph Research Analytical, modelo DDM 2911).

\subsection{Determinação do pH, sólidos solúveis e açúcares redutores e totais}

As determinações do pH, sólidos solúveis, açúcares redutores e totais foram realizadas através dos métodos descritos pelo Instituto Adolfo Lutz (1985).

\subsection{Metodologia para análise dos resultados}

A metodologia empregada para o cálculo dos fatores (produtividade volumétrica (Qp), rendimento em etanol (Yp/s) e tempo de geração (Tg)) foi proposta por Borzani (1986) e Moser (1988). As velocidades específicas de crescimento celular, de consumo de extrato aparente e de produção de etanol foram calculadas utilizando as equações do método geométrico de cálculo de derivadas proposto por Le Duy e Zajic (1973).

\subsection{Metodologia para análise sensorial}

A análise sensorial da cerveja produzida foi realizada através do teste afetivo de aceitação com escala hedônica estruturada com 9 pontos. Os testes foram realizados em cabines de análise sensorial, no Laboratório de Análise Sensorial do Departamento de Tecnologia da Universidade Estadual de Feira de Santana / BA. Os testes foram conduzidos com 60 provadores não selecionados e não treinados, do público universitário, de ambos os sexos e maiores de 18 anos. Além da aceitação, ainda foi avaliado a intenção de compra dos avaliadores diante do novo produto.

\section{RESULTADOS E DISCUSSÃO}

\subsection{Estudo cinético da fermentação}

Através das representações gráficas da concentração celular obtida pela contagem celular em câmara de Neubaur, Figura 1, e por peso celular, Figura 2, observa-se que a levedura alcançou máxima concentração celular após 34 horas de fermentação, 2,2×10 8 (cel/mL) pelo método de viabilidade celular e 8,31 g/L através do peso seco. Valores semelhantes de células em suspensão foram encontrados por Carvalho et al. (2008) na fermentação de mosto utilizando banana como adjunto, a 15 ${ }^{\circ} \mathrm{C}$, com extrato aparente inicial de $12{ }^{\circ} \mathrm{P}$. Após as 34 horas iniciais da fermentação a levedura começa a entrar na fase de invibialização celular (ou fase de morte). Observa-se que a fase de morte da 
levedura é iniciada no momento em que a temperatura do processo é reduzida até $0{ }^{\circ} \mathrm{C}$, iniciando a maturação da cerveja, demonstrando a relação direta entre o crescimento celular e a temperatura.

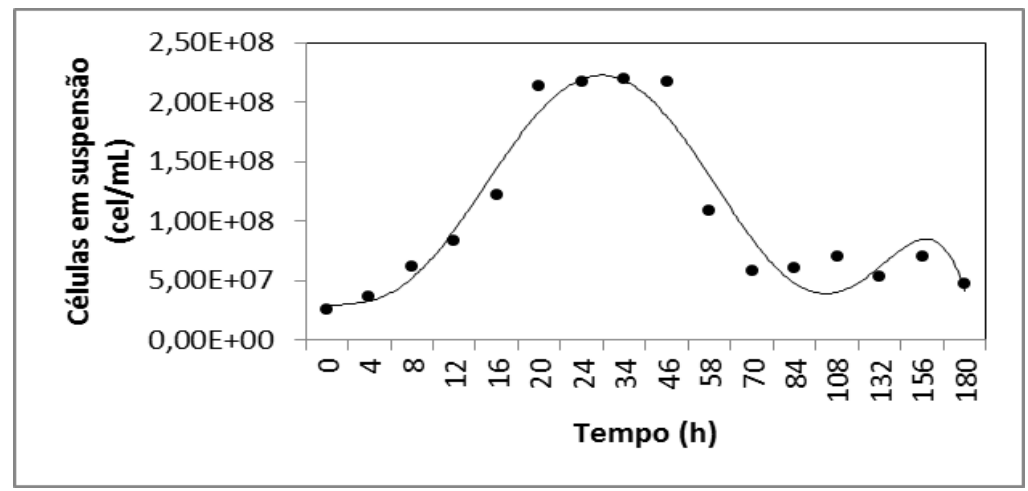

Figura 1 - Células em suspensão (cel/mL).

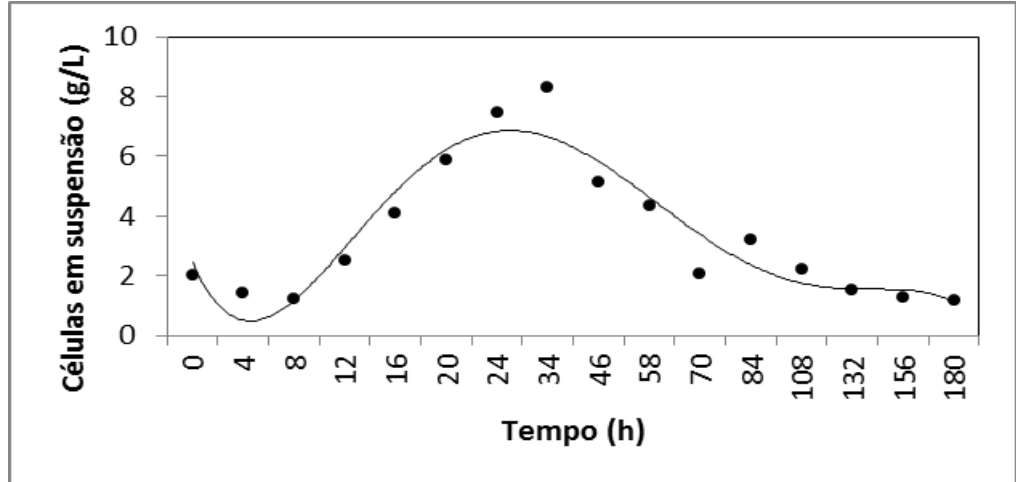

Figura 2 - Células em suspensão (g/L).

A Figura 3 apresenta a evolução da concentração celular em relação a redução da densidade durante o processo de fermentação primária e secundária da cerveja. Observa-se que nas primeiras 8 horas de fermentação há uma redução na quantidade de células de levedura presentes no meio, esse fenômeno pode ser explicado através da floculação celular (GUIDO, 2004). Após esse período é observado um rápido crescimento celular, característica da fase log ou exponencial até o ponto máximo de concentração $(8,31 \mathrm{~g} / \mathrm{L})$ seguido pela fase de declínio celular. Observa-se que durante todo o período da fermentação há declínio da densidade inicial do mosto e no ponto final do processo a redução chega a ser de $2,35 \%$. 


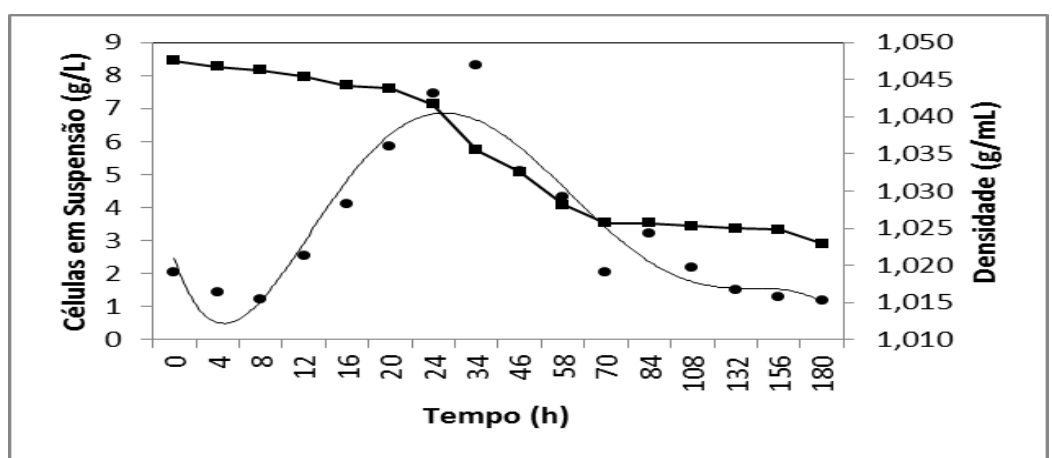

Figura 3 - Células em suspensão $(\mathrm{g} / \mathrm{L})(\bullet)$ x Densidade $(\mathrm{g} / \mathrm{mL})(\bullet)$.

A Figura 4 apresenta as velocidades específicas de crescimento de células em suspensão, consumo de substrato (açúcares totais) e produção de etanol em função do tempo, para a cerveja produzida. As velocidades foram calculadas através do método geométrico de derivadas proposto por Le Duy e Zajic (1973). Observa-se que o perfil da velocidade de consumo de substrato $\mu_{\mathrm{s}}$ apresentou semelhança ao perfil da velocidade mostrado para a produção de etanol $\mu_{\mathrm{p}}$, identificando a relação direta entre esses dois parâmetros. Observa-se também que os valores de $\mu_{\mathrm{x}}, \mu_{\mathrm{s}}$ e $\mu_{\mathrm{p}}$ atingiram seus pontos máximos $\left(0,134 \mathrm{~h}^{-1}, 2,95 \mathrm{~g}_{\mathrm{s}} / \mathrm{g}_{\mathrm{x}} \cdot \mathrm{h}\right.$ e $\left.1,00 \mathrm{~g}_{\mathrm{p}} / \mathrm{g}_{\mathrm{x}} \cdot \mathrm{h}\right)$ nos tempos de $8,4 \mathrm{e} 4$ horas, respectivamente. A partir do valor máximo da velocidade específica de crescimento celular $\left(\mu_{\mathrm{xmáx}}\right)$ pôde-se calcular o tempo de geração $\left(\mathrm{t}_{\mathrm{g}}\right)$ da levedura que foi de 5,17 horas.

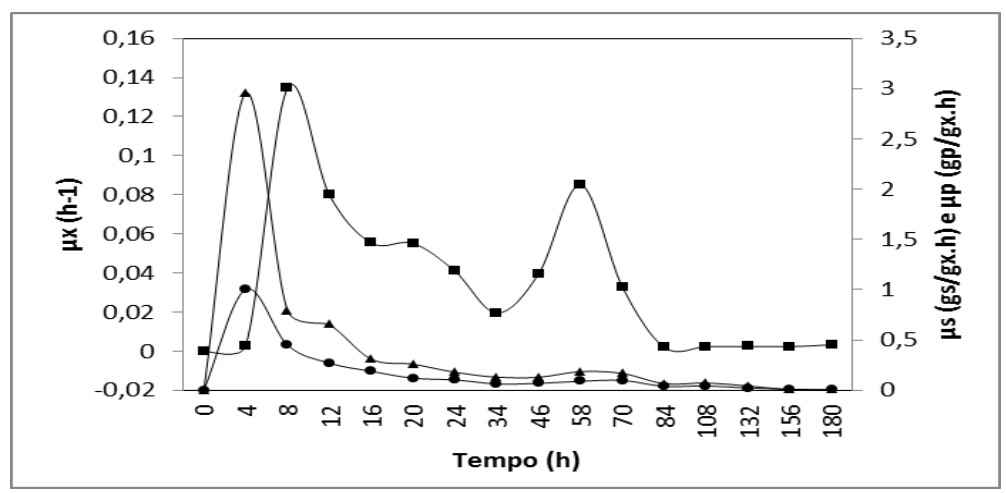

Figura 4 - Velocidade específica de crescimento celular $\left(\mu \mathrm{x}^{\bullet}\right)$, consumo de extrato aparente $(\mu \mathrm{\Delta} \boldsymbol{\Delta})$, e produção de etanol ( $\left.\mu p^{\bullet}\right)$, para a cerveja produzida.

\subsection{Consumo de extrato aparente e produção de etanol}

A Figura 5 apresenta o consumo de extrato aparente e a produção de etanol durante o tempo de fermentação. No ponto final da fermentação primária, após 34 horas, a $15{ }^{\circ} \mathrm{C}$, observou-se a produção de 27,54 g/L de etanol, o equivalente a 3,39 \% v/v, com o consumo de 54,22 g/L de extrato aparente. Observa-se que a leveduras continuam seu metabolismo de produção de etanol durante a fermentação secundária, mesmo em temperatura reduzida de $0{ }^{\circ} \mathrm{C}$. Ao final de 180 horas de processo a cerveja apresentou 43,47 g/L de etanol, 5,51\% v/v, com consumo de 97,01 g/L de extrato aparente, representando grau de fermentação, ou fermentabilidade, de 73,49\%, valor um pouco abaixo do percentual sugerido por Tschope (2001) de 80\% para cervejas do tipo Pilsen. 
Não foi observado valores crescentes no fator de conversão como a produtividade volumétrica (Qp) e o rendimento real em etanol (Yp/s), Figura 6, como descrito por Hiss (2001). A produtividade volumétrica em etanol apresentou variação durante todo o processo fermentativo alcançando seu valor máximo de 1,07 g/L.h em 12 horas de fermentação a $15^{\circ} \mathrm{C}$ com redução desse valor após esse ponto. $\mathrm{O}$ maior valor de rendimento real em etanol (Yp/s) foi alcançado na fase de fermentação secundária da cerveja em 50 horas após a inoculação da levedura. Esse valor foi de 0,50 g/g, sendo muito próximo do valor ao teórico proposto por Gay-Lussac $(0,511 \mathrm{~g} / \mathrm{g})$.

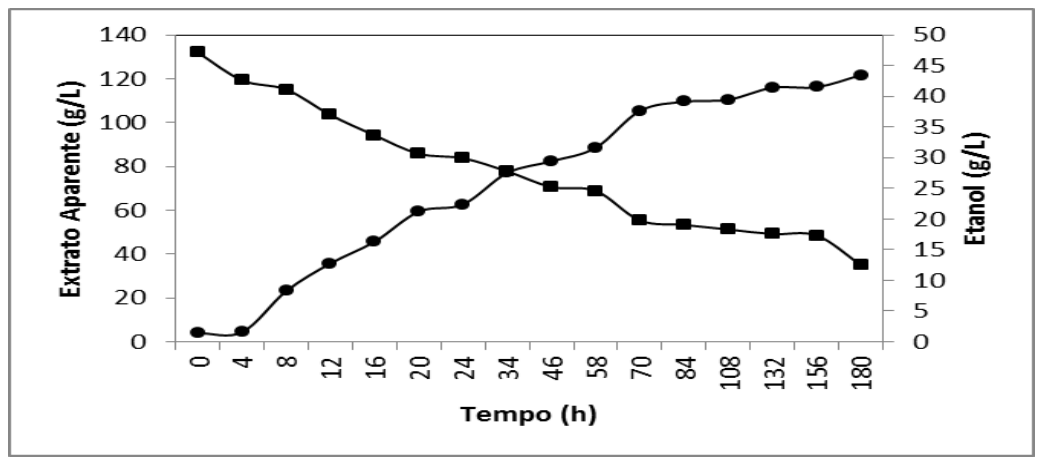

Figura 5 - Consumo de extrato aparente $(\mathrm{g} / \mathrm{L})(\cdot)$ x produção de etanol $(\mathrm{g} / \mathrm{L})(\bullet)$.

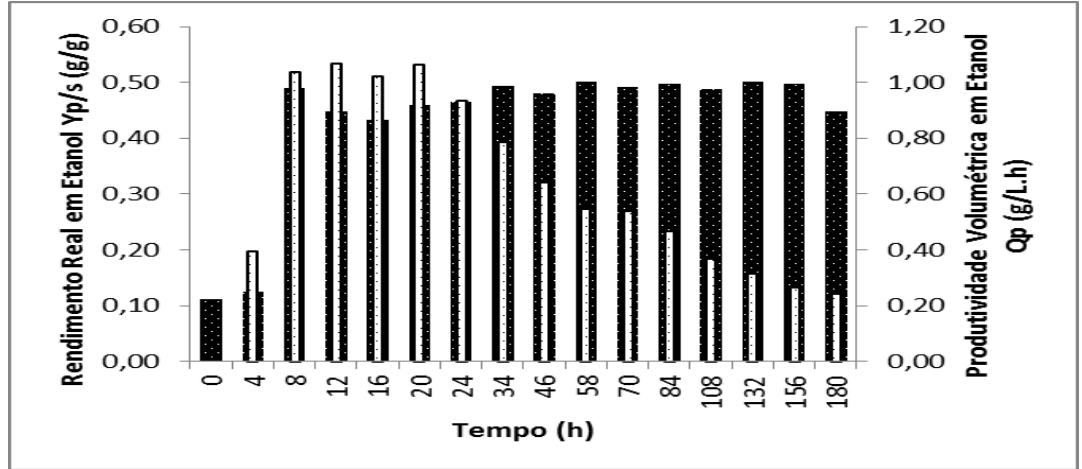

Figura 6 - Valores de Rendimento real em etanol (Yp/s) (g/g) (•) e Produtividade volumétrica em etanol (Qp) (g/L.h) (口).

\subsection{Análise sensorial}

O teste sensorial da cerveja produzida foi realizado para avaliar a aceitação do produto e a intenção de compra diante do consumidor. Para isso, o teste afetivo de aceitação com escala hedônica estruturada com 9 ponto foi realizado. No teste também foi avaliada a intenção de compra do produto através de escala constituída com 5 pontos, variando entre "certamente compraria" e "certamente não compraria". As amostras foram retiradas diretamente do tanque de fermentação/maturação e servidas aos provadores em copos de polietileno de $250 \mathrm{~mL}, 100 \mathrm{~mL}$ de cerveja na temperatura de aproximadamente $5{ }^{\circ} \mathrm{C}$, acompanhadas de água mineral, torrada e patê de presunto.

Observou-se que a cerveja teve boa aceitação diante dos provadores, ficando o produto com 85\% de aceitação entre "gostei" e "gostei muitíssimo". Quanto a intenção de compra 87\% dos provadores indicaram interesse na compra do produto, indicados entre "compraria" e "certamente 
compraria". Os principais comentários dos provadores foram em relação a cor, ao aroma e ao sabor da cerveja. A maioria dos provadores indicou que a cerveja apresentava cor mais escura, em comparação às cervejas comerciais, além de ter turbidez acentuada devido a não utilização da clarificação no processo. Os provadores indicaram a presença de "aroma de cajá" e sabor perceptível da fruta na cerveja analisada, fator positivo na avaliação.

A cerveja tipo Lager com suco misto de cajá e sacarose como adjunto do malte pode ser observada na Figura 7.

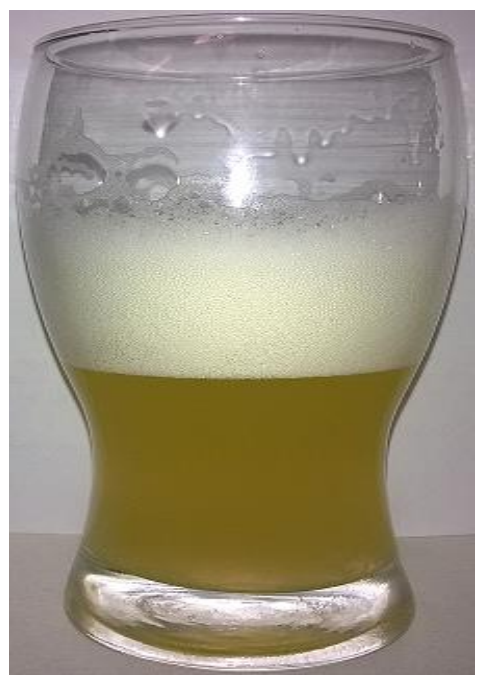

Figura 7 - Cerveja com suco de cajá tipo Lager, teor alcoólico de 5,51\% v/v.

\section{CONCLUSÕES}

Os resultados obtidos das análises da cerveja produzida em planta piloto de 60 litros, mostraram a viabilidade físico-química e sensorial do produto. A cerveja apresentou 5,51\% de etanol (v/v), valor muito próximo do teor de álcool encontrado em cervejas comerciais. A fermentabilidade de 73,49\% mostra que houve boa utilização do extrato aparente inicial na formação de produtos pela levedura, isso pode ser também comprovado pelos valores de rendimento real em etanol durante todo o processo, chegando a atingir o valor de $0,50 \mathrm{~g} / \mathrm{g}$ após 50 horas de fermentação. A análise sensorial indicou a boa aceitabilidade do novo produto diante dos analisadores com $85 \%$ de aprovação da cerveja, sendo que $87 \%$ dos 60 provadores demonstraram interesse na compra da cerveja.

\section{REFERÊNCIAS}

ASBC (1996).- Methods of Analysis of the American Society of Brewing Chemists. Eighth Revised Edition. Minnesota: The Technical Committee and the Editorial Committe of the ASBC.

BORZANI, W. (1986). Cinética de processos fermentativos. Revista Brasileira de Engenharia, v.3, n.2, p.61.

CARVAlHO, G. B. M.; SILVA, D. P.; BENTO, C. V.; VICENTE, A. A., TEIXEIRA, J. A.; FELIPE, M. G. A.; ALMEIDA E SILVA, J. B. (2008). Banana as adjunct in beer production: Applicability and 
performance of fermentative parameters. Applied Biochemistry and Biotechnology, 155, 53-62. doi: 10.1007/s12010-008-8458-y.

CATALUÑA, E. (1988). As uvas e os vinhos. 2.ed. Rio de Janeiro: Globo, 207p.

GUIDO, L. F., RODRIGUES, P. G., RODRIGUES, J. A., GONCALVES, C. R., \& BARROS, A. A. (2004). Food Chemistry, 87, 187-193. doi: 10.1016/j.foodchem.2003.10.033.

HISS, H. Cinética de Processos Fermentativos. (2001) In: ALMEIDA LIMA, U.; AQUARONE, E.; BORZANI, W.; SCHMIDELL, W. Biotecnologia Industrial. São Paulo: Edgard Blücher, v. 2. p. 93 122.

INSTITUTO ADOLFO LUTZ. (1985). Normas analíticas do Instituto Adolfo Lutz: métodos químicos e físicos para análise de alimentos. 3 ed. São Paulo: O Instituto, v.1, 533 p.

LE DUY, A.; ZAJIC, J. E. (1973). A Geometrical Approach for Differentiation of an Experimental Function at a Point: Applied to Growth and Product Formation. Biotechnology and Bioengineering, New York, v. 15, 805-815.

MOSER, A. (1988). Bioprocess Technology. Kinetics and Reactors. New York: Springer-Verlag, $451 \mathrm{p}$.

TSCHOPE, E. C.(2001). Microcervejarias e Cervejarias. A Historia, a Arte e a Tecnologia. São Paulo: Editora Aden, 223 p.

VENTURINI FILHO, W. G.; CEREDA, M. P. Cerveja. in: Almeida Lima, U.; Aquarone, E.; Borzani, W.; Schmidell, W. (2001). Biotecnologia Industrial. Volume 4. Biotecnologia na Produção de Alimentos. São Paulo: Edgar Blucher, 91-144. 